\title{
Rapid infusion rituximab is well tolerated in patients with primary CNS lymphoma
}

\author{
Lisa Modelevsky*,1, Richard Tizon¹, Samantha N Reiss ${ }^{1}$ Marcel Smith¹, Rachel Garonce ${ }^{1}$ \& \\ Thomas Kaley ${ }^{1}$ \\ ${ }^{1}$ Memorial Sloan Kettering Cancer Center, 1275 York Ave, New York, NY 10065, USA \\ *Author for correspondence: Tel.: +1 212639 7067; Fax: +1 212639 2171; modelevl@mskcc.org
}

\begin{abstract}
Aim: To establish the safety and feasibility of rapidly infusing rituximab over $90 \mathrm{~min}$ in patients with primary CNS lymphoma (PCNSL). Patients \& methods: We retrospectively reviewed all patients with PCNSL who received rapid rituximab infusions (RRI) from January 2016 to January 2017. Primary end point was incidence of infusion reactions. Results \& conclusion: 11 patients received a total of 44 RRIs. Rituximab was dosed at 500 or $750 \mathrm{mg} / \mathrm{m}^{2}$. Premedication included acetaminophen and diphenhydramine. No infusion reactions occurred during any RRI. Two infusions were administered with steroids for neurologic symptoms at baseline (4.5\%). Rapid administration of rituximab was safe and feasible for patients with PCNSL and at the higher doses received.
\end{abstract}

First draft submitted: 23 January 2018; Accepted for publication: 25 May 2018; Published online: 17 September 2018

Keywords: neuro-oncology • primary CNS lymphoma • rapid infusion • rituximab

Rituximab is a chimeric monoclonal antibody targeted against the CD20 antigen on B-lymphocytes. It is currently US FDA approved for treating non-Hodgkin's lymphoma (NHL), chronic lymphocytic leukemia (CLL), rheumatoid arthritis, granulomatosis with polyangiitis, and microscopic polyangiitis [1]. The addition of rituximab to standard chemotherapy has been shown to significantly improve overall survival for patients with systemic diffuse large B-cell lymphoma (DLBCL) [2-4]. In light of this data, rituximab was studied in combination with standard chemotherapy for primary CNS lymphoma (PCNSL) given that the histologic subtype in more than $90 \%$ of cases is DLBCL [5]. Based on the encouraging results from several studies, rituximab has been incorporated into the majority of standard induction regimens for CD20-positive PCNSL [6-16]. Today, upfront treatment of PCNSL primarily consists of induction chemoimmunotherapy with rituximab and methotrexate-based regimens (most commonly methotrexate, cytarabine, thiotepa, rituximab [MATRix], or rituximab, methotrexate, vincristine, procarbazine [RMVP], or methotrexate, temozolomide, rituximab [MTR]) $[7,8,13,14,17,18]$. In these regimens, rituximab is dosed between 375 and $500 \mathrm{mg} / \mathrm{m}^{2}$. Induction therapy is then generally followed by consolidation with high-dose chemotherapy, whole-brain radiotherapy or high-dose chemotherapy in combination with autologous stem cell transplant $[7,18]$. Rituximab has also been studied in the maintenance setting for patients with PCNSL unable to tolerate more aggressive consolidation. In the study conducted by Ney et al., maintenance rituximab was dosed at $750 \mathrm{mg} / \mathrm{m}^{2}$ and administered every $1-3$ months [19].

Rituximab is generally well tolerated, though infusion-related reactions (IRRs) are common. At standard infusion rates, grade 3 and 4 IRRs occur in approximately 7\% of NHL and CLL patients during the first infusion, 2\% during fourth infusion, and $0 \%$ during the eighth infusion [1]. These reactions may range from fever, chills, rigors and hypotension to more severe reactions including pulmonary events, urticaria, anaphylaxis or death [1]. To minimize the risk of IRRs, the FDA-approved label recommends premedicating patients with acetaminophen and an antihistamine. The first infusion is initiated at a rate of $50 \mathrm{mg} / \mathrm{h}$. In the absence of an IRR, the infusion rate is then increased by increments of $50 \mathrm{mg} / \mathrm{h}$ every $30 \mathrm{~min}$ to a maximum of $400 \mathrm{mg} / \mathrm{h}$. Subsequent infusions are initiated at $100 \mathrm{mg} / \mathrm{h}$ and in the absence of IRR increased by $100 \mathrm{mg} / \mathrm{h}$ every $30 \mathrm{~min}$ to the same maximum rate. This labor-intensive process averages $4-5 \mathrm{~h}$ for the first infusion and $3 \mathrm{~h}$ for subsequent infusions. To reduce patient inconvenience and the burden on healthcare resources, a 90-min infusion schedule was previously developed and analyzed for safety and tolerability in patients with systemic lymphoma [20]. The results of a Phase III rapid 
infusion study led to the FDA's approval of a 90-min infusion in 2012. This study was conducted in 363 patients with previously untreated systemic NHL receiving rituximab dosed at $375 \mathrm{mg} / \mathrm{m}^{2}$ as part of standard treatment with rituximab, cyclophosphamide, doxorubicin, vincristine, and prednisone or rituximab, cyclophosphamide, vincristine, and prednisone. Patients who did not experience a grade 3 or 4 IRR during cycle 1 were eligible for a 90-min infusion during cycles $2-8$. The incidence of grade 3 or 4 IRRs during cycles $2-8$ was $2.8 \%$ [21]. Comparable rates of IRRs have been reported in studies administering rituximab at both standard infusion rates and rapid infusion rates [20,22-24]. However, RRI studies thus far have not included patients with CNS lymphoma or the higher doses these patients may receive for improved brain penetration ranging from 500 to $750 \mathrm{mg} / \mathrm{m}^{2}$.

Patients with systemic B-cell mediated diseases being treated at our institution have been receiving RRI for several years. Though we did not foresee any clinical issues with administering RRIs to patients with PCNSL, these patients were initially excluded from this method of administration given the lack of safety data in this population and the higher doses they receive. To standardize administration, and to improve both resource utilization and patient satisfaction, our chemotherapy committee recently approved the expansion of RRI to patients with PCNSL. We sought to identify all patients with PCNSL who had received RRI and confirm if rapid infusion of rituximab over 90 min was both safe and feasible in these patients and at the higher doses they may receive.

\section{Methods}

Study design

This study was an Institutional Review Board-approved, single-center, retrospective chart review assessing patients with PCNSL who received RRI from the time of implementation in this population in January 2016 and until 1 year later in January 2017. The primary end point of this study was incidence of infusion reactions of any grade during RRI.

\section{Rapid rituximab infusion practice for PCNSL}

Eligibility criteria for patients with PCNSL to receive RRI included age greater than 18 years, previous standard rate rituximab infusion(s) without infusion reaction of any grade and patients were not receiving chemotherapy on the same day. Concomitant corticosteroids were permitted in order to control disease-related neurologic symptoms. If chemotherapy was part of treatment, rituximab was administered the day prior.

Patients who met criteria for RRI received the first $50 \mathrm{ml}$ of the infusion $(\sim 20 \%$ of total volume) over $30 \mathrm{~m}$ and the remaining volume over $1 \mathrm{~h}$. Premedication was administered $30 \mathrm{~min}$ prior to infusion and consisted of acetaminophen $650 \mathrm{mg}$ orally and diphenhydramine $25 \mathrm{mg}$ intravenously. Rituximab was diluted in normal saline to a concentration of $\leq 4 \mathrm{mg} / \mathrm{ml}$. Patients were monitored during RRI for signs of infusion reaction and vitals were measured after completion of infusion. If an IRR occurred, the infusion would be held until symptoms improved and then resumed at one-half of the rate at which reaction occurred. In the absence of IRR, patients continued to receive RRI for their remaining cycles.

\section{Data analysis}

Patient information was reviewed from pharmacy databases and medical records. IRRs were characterized according to the Common Terminology Criteria for Adverse Events version 4.03 [25]. Descriptive statistics were implemented to describe patient characteristics and incidence of IRRs.

\section{Results}

From January 2016 to January 2017, 17 patients with PCNSL were screened for RRI eligibility; six were excluded due to previous IRR. 11 patients (65\%) were eligible for RRI and received a total of 44 RRIs over the 1 year period. The majority of patients had a histologic subtype of DLBCL (91\%) and two patients (18\%) had leptomeningeal disease. All patients had a normal absolute lymphocyte count, and median Karnofsky Performance Status was 90. Lactate dehydrogenase (LDH) was available for seven patients and was elevated in three of these patients prior to the first RRI. Baseline characteristics for patients who received RRI are provided in Table 1.

Patients received RRI either in conjunction with chemotherapy (73\%) or as single-agent maintenance therapy (27\%). The majority of patients received rituximab as part of RMVP which includes rituximab dosed at $500 \mathrm{mg} / \mathrm{m}^{2}$ on day 1 , methotrexate $3.5 \mathrm{~g} / \mathrm{m}^{2}$ and vincristine $1.4 \mathrm{mg} / \mathrm{m}^{2}$ on day 2 , all administered every 14 days for up to 8 cycles with the addition of procarbazine $100 \mathrm{mg} / \mathrm{m}^{2} / \mathrm{d}$ on days 1 through 7 of odd cycles (64\%). Ten patients received rituximab dosed at $500 \mathrm{mg} / \mathrm{m}^{2}$ and one patient received $750 \mathrm{mg} / \mathrm{m}^{2}$; doses were not capped. The median 
Table 1. Baseline patient characteristics.

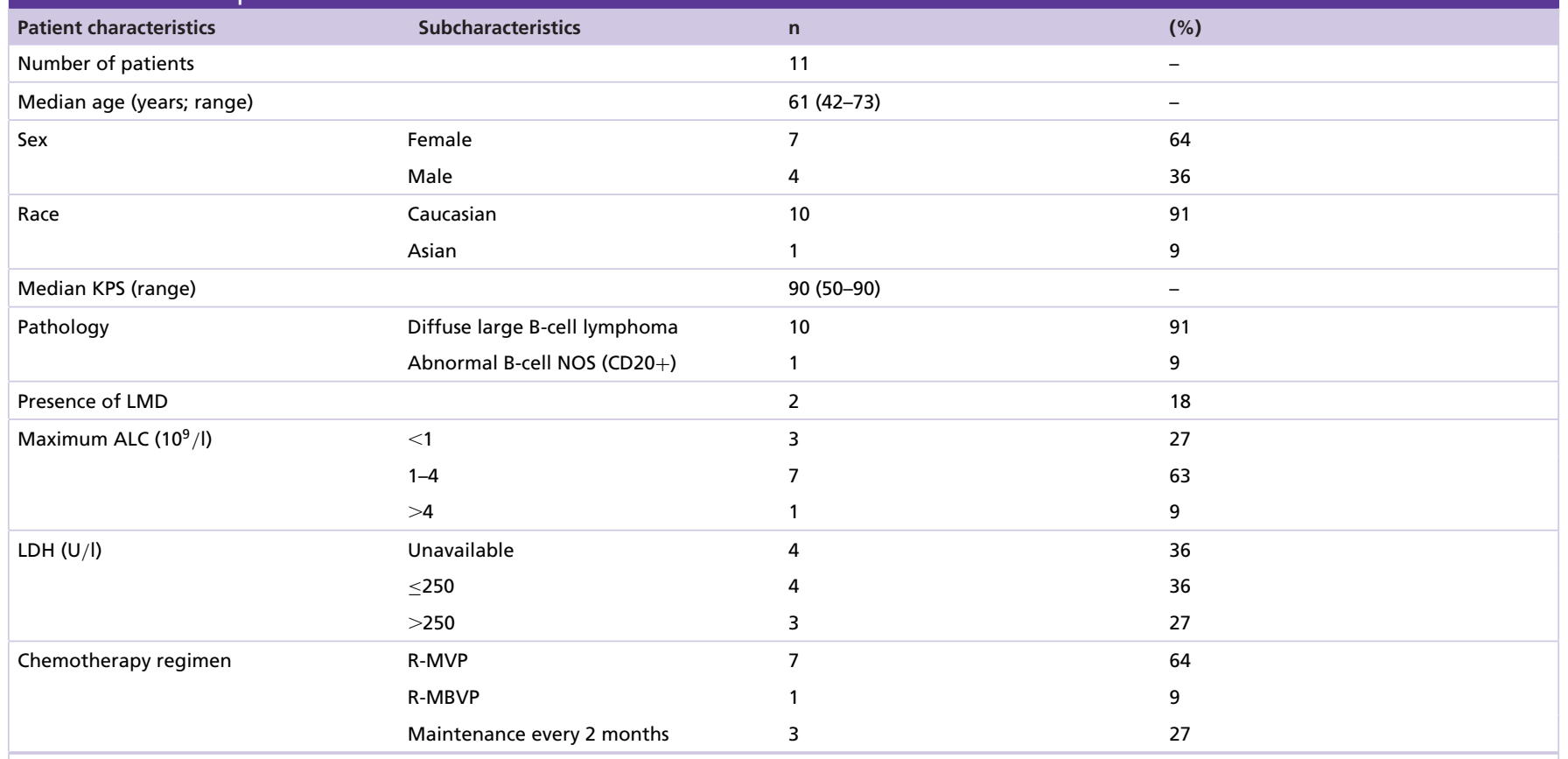

ALC: Absolute lymphocyte count; KPS, Karnofsky performance status; LDH: Lactate dehydrogenase; LMD: Leptomeningeal disease; NOS: Not otherwise specified; R-MBVP: Rituximab, methotrexate, carmustine, etoposide, prednisone; R-MVP: Rituximab, methotrexate, vincristine, procarbazine.

Table 2. Rapid rituximab infusion characteristics.

\begin{tabular}{|c|c|c|}
\hline Characteristics & $\mathbf{n}$ & $(\%)$ \\
\hline Total number of rapid infusions & 44 & 100 \\
\hline Median number of infusions per patient (range) & 4 & $(1-7)$ \\
\hline Number of infusion reactions during rapid & 0 & 0 \\
\hline Median rituximab dose in milligrams (range) & 950 & $(750-1500)$ \\
\hline Patients receiving 1 standard infusion prior to rapid & 7 & 64 \\
\hline Patients receiving $\geq 2$ standard infusions prior to rapid & 4 & 36 \\
\hline Number of rapid infusions with concurrent steroids & 2 & 5 \\
\hline
\end{tabular}

number of RRIs per patient was four (range 1-7) since most patients had already received more than one of their planned rituximab infusions at standard rate prior to expansion of RRI to PCNSL patients. The median dosage received was $950 \mathrm{mg}$ (range, 750-1500 mg). Characteristics of RRIs are provided in Table 2.

All patients received standard premedication with acetaminophen and diphenhydramine. Two patients had been receiving dexamethasone $2 \mathrm{mg}$ daily during the first RRI for disease-related neurologic symptoms; 42 of 44 RRIs (95\%) were administered without steroids. All patients continued receiving RRI for entire duration of rituximab therapy.

Prior to the first RRI, seven out of $11(64 \%)$ patients had received one standard infusion, one out of 11 (9\%) had received three, and three out of the $11(27 \%)$ patients had been receiving single-agent maintenance rituximab every 2 months at standard infusion rate for more than 2 years. No infusion reactions of any grade were observed during the 44 RRIs.

\section{Discussion}

Rapid rituximab infusions were safe and feasible for all patients in this cohort. Patients did not experience IRRs whether they received RRI as part of chemoimmunotherapy or as single-agent maintenance therapy every 2 months. All but two infusions were administered without concomitant corticosteroids. Previous studies have also shown that RRIs are tolerated by patients whether administered with or without steroids [20,26]. 
Our practice differed from others in that only patients without an IRR during standard rate rituximab infusion(s) met criteria for RRI. This is different from the prescribing information which states that patients who experience a grade 1 or 2 reaction during cycle 1 may receive 90-min infusions starting with cycle 2 . Our rationale for not administering RRI subsequent to an IRR was based on the lack of safety data for using RRI in this population and within this dose range. It is likely, however, that patients with PCNSL who experience grade 1 or 2 reactions during cycle 1 would tolerate RRIs with a low incidence of IRRs especially since these patients do not have systemic disease and do not have elevated absolute lymphocyte counts; a known predictor for IRR [27]. Our study also differed in that LDH was not available for all patients. We do not see this as a limitation since LDH is not routinely monitored for patients with PCNSL given the absence of systemic disease. Additionally, the doses of rituximab our patients received ranging from $500-750 \mathrm{mg} / \mathrm{m}^{2}$ were higher than those included in previous rapid rituximab studies for systemic NHL. Furthermore, the $750 \mathrm{mg} / \mathrm{m}^{2}$ dose is higher than FDA approved doses. It is important to note that these higher doses of rituximab used for PCNSL are considerably costlier than the standard doses used for systemic lymphoma, however, these higher doses were previously incorporated into studies primarily based on CSF pharmacokinetics. For patients with PCNSL, the efficacy of rituximab dosed at $375 \mathrm{mg} / \mathrm{m}^{2}$ has not been compared directly with $500 \mathrm{mg} / \mathrm{m}^{2}$ for induction. Lower dosages would afford cost savings although it is unknown if reducing the dosage would decrease efficacy.

The small sample size of our study is a limitation. However, given the rarity of PCNSL with an overall incidence rate of 0.45 per 100,000 persons, this study provides much-needed data to support the expansion of RRI to PCNSL patients receiving rituximab in the outpatient setting [28]. Based on the results of this study, patients with PCNSL who tolerate their first rituximab infusion administered at the standard rate may receive RRI starting with their second infusion. Patients who previously received more than one standard rituximab infusion and tolerated all prior infusions at standard rate may also be offered RRI.

Rapidly infusing rituximab over $90 \mathrm{~min}$ has been shown to save an average of $10.2 \mathrm{~h}$ per patient over the course of rituximab treatment, thereby improving patient satisfaction and offering an economic advantage by reducing chair time, resource utilization, and nursing monitoring. Additionally, patients with CNS disease were excluded from subcutaneous rituximab studies and it is unknown if this route of administration affects CNS penetration [29,30]. Therefore, this study provides essential safety and feasibility data supporting the expansion of RRIs to patients with PCNSL in an effort to decrease financial impact and improve patient satisfaction.

\section{Future perspective}

Rituximab has become a standard component of treatment regimens for PCNSL. Most recently, the IELSG32 trial showed that the addition of rituximab to high-dose methotrexate and thiotepa improved overall response rates from 53 to $73 \%$ and improved the overall 2-year progression free survival from 36 to $46 \%$ [8]. Undoubtedly, rituximab will continue to be included in standard induction regimens unless in the years to come other treatments prove superior. CSF levels of rituximab after intravenous administration are less than $1 \%$ of corresponding serum levels. As intravenous dosage increases, CSF concentration increases though it is unknown if higher concentration improves clinical outcomes [31]. Comparing the efficacy of lower versus higher doses of rituximab for the treatment of PCNSL may have further cost saving implications. An additional area of research that could further reduce healthcare costs and potentially improve patient satisfaction would be to determine if the efficacy of subcutaneously administered rituximab is comparable with intravenous administration for the treatment of PCNSL. Subcutaneous administration of rituximab results in lower peak serum concentrations versus intravenous administration $[32,33]$. Therefore, it is possible that subcutaneous administration may impair the penetration of rituximab across the blood-brain barrier; research is warranted before expanding this method of administration to all patients with PCNSL. 
Summary points

- The safety and feasibility of rapidly infusing rituximab over 90 min has previously been established in patients with systemic B-cell lymphomas.

- Patients with CNS lymphoma were excluded from rapid infusion studies.

- Given the lack of data with rapid infusion in patients with CNS lymphoma, it was previously unknown if this faster rate of administration was also safe and feasible in this population.

- We retrospectively reviewed all patients with primary CNS lymphoma (PCNSL) who received rapid rituximab infusion over 90 min within a 1 year period.

- A total of 44 rapid infusions at doses of $500-750 \mathrm{mg} / \mathrm{m}^{2}$ were administered to this population.

- Patients received standard premedication with acetaminophen and diphenhydramine.

- Only two infusions were administered with steroids which were indicated for neurologic symptoms at baseline.

- No infusion reactions of any grade were experienced by patients with PCNSL.

- This study supports the expansion of rapid infusion rituximab to patients with PCNSL and at the higher dosages received affording an economic advantage over standard infusion times.

- Subcutaneous administration cannot currently be recommended for this population due to the lack of efficacy data in patients with PCNSL.

\section{Disclosure}

The authors state that they have obtained appropriate institutional review board approval or have followed the principles outlined in the Declaration of Helsinki for all human or animal experimental investigations.

\section{Open access}

This work is licensed under the Creative Commons Attribution 4.0 License. To view a copy of this license, visit http://creativecomm ons.org/licenses/by/4.0

\section{References}

Papers of special note have been highlighted as: $\bullet$ of interest; $\bullet \bullet$ of considerable interest

1. Rituximab [product monograph]. Hoffman-La Roche, Mississauga, ON, Canada. (February 22, 2017) (2016). www.rochecanada.com/content/dam/roche_canada/en_CA/documents/Research/ClinicalTrialsForms/Products/ConsumerInformation/ MonographsandPublicAdvisories/Rituxan/RituxanIV_PM_E.pdf

2. Coiffier B, Thieblemont C, Van Den Neste E et al. Long-term outcome of patients in the LNH-98.5 trial, the first randomized study comparing rituximab-CHOP with standard CHOP chemotherapy in DLBCL patients: a study by the Groupe d'Etudes des Lymphomes de l'Adulte. Blood 116(12), 2040-2045 (2010).

3. Pfreundschuh M, Kuhnt E, Trumper L et al. CHOP-like chemotherapy with or without rituximab in young patients with good-prognosis diffuse large-B-cell lymphoma: 6-year results of an open-label randomized study of the MabThera International Trial (MInT) Group. Lancet Oncol. 12(11), 1013-1022 (2011).

4. Habermann TM, Weller EA, Morrison VA et al. Rituximab-CHOP versus CHOP alone or with maintenance rituximab in older patients with diffuse large B-cell lymphoma. J. Clin. Oncol. 24(19), 3121-3127 (2006).

5. Rubenstein J, Ferreri AJ, Pittaluga S. Primary lymphoma of the central nervous system: epidemiology, pathology and current approaches to diagnosis, prognosis and treatment. Leuk. Lymphoma 49(Suppl. 1), 43-51 (2008).

6. Shah GD, Yahalom J, Correa DD et al. Combined immunochemotherapy with reduced whole-brain radiotherapy for newly diagnosed primary CNS lymphoma. J. Clin. Oncol. 25(30), 4730-4735 (2007).

7. Kerbauy MN, Moraes FY, Lok BH et al. Challenges and opportunities in primary CNS lymphoma: a systematic review. Radiother. Oncol. 122(3), 352-361 (2017).

- Provides an overview of current standards of practice for patients with primary CNS lymphoma; it includes a section on the role of rituximab.

8. Ferreri AJ, Cwynarski K, Pulczynski E et al. Chemoimmunotherapy with methotrexate, cytarabine, thiotepa, and rituximab (MATRix regimen) in patients with primary CNS lymphoma: results of the first randomization of the International Extranodal Lymphoma Study Group-32 (IELSG32) Phase II trial. Lancet Haematol. 3(5), e217-e227 (2016).

9. Holdhoff M, Ambady P, Abdelaziz A et al. High-dose methotrexate with or without rituximab in newly diagnosed primary CNS lymphoma. Neurology 83(3), 235-239 (2014).

10. Zhao HT, Chen J, Shi SB, Tian J, Tao RJ. Pemetrexed plus rituximab as second-line treatment for primary central nervous system lymphoma. Med. Oncol. 32(1), 351 (2015). 
11. Mappa S, Marturano E, Licata G et al. Salvage chemoimmunotherapy with rituximab, ifosfamide and etoposide (R-IE regimen) in patients with primary CNS lymphoma relapsed or refractory to high-dose methotrexate-based chemotherapy. Hematol. Oncol. 31(3), $143-150$ (2013).

12. National Comprehensive Cancer Network. Central Nervous System Cancers (Version 1.2016) (2017 March 13) (2016).www.nccn.org/professionals/physician_gls/pdf/cns.pdf

13. Omuro A, Correa DD, Deangelis LM et al. R-MPV followed by high-dose chemotherapy with TBC and autologous stem-cell transplant for newly diagnosed primary CNS lymphoma. Blood 125(9), 1403-1410 (2015).

- Investigates R-MPV: the regimen the majority of the patients received in our study. In this regimen, rituximab is dosed at $500 \mathrm{mg} / \mathrm{m}^{2}$ which is higher than dosages seen in some of the other primary CNS lymphoma regimens.

14. Rubenstein JL, Hsi ED, Johnson JL et al. Intensive chemotherapy and immunotherapy in patients with newly diagnosed primary CNS lymphoma: CALGB 50202 (Alliance 50202). J. Clin. Oncol. 31(25), 3061-3068 (2013).

15. Nayak L, Abrey LE, Drappatz J et al. Multicenter Phase II study of rituximab and temozolomide in recurrent primary central nervous system lymphoma. Leuk. Lymphoma 54(1), 58-61 (2013).

16. Chamberlain MC, Johnston SK. High-dose methotrexate and rituximab with deferred radiotherapy for newly diagnosed primary B-cell CNS lymphoma. Neuro-oncology 12(7), 736-744 (2010).

17. Fabbri A, Cencini E, Cerase A, Bocchia M. New perspectives for patients with primary CNS lymphoma. Radiother. Oncol. 124(2), 331-332 (2017).

18. Grommes C, Deangelis LM. Primary CNS Lymphoma. J. Clin. Oncol. 35(21), 2410-2418 (2017).

19. Ney DE, Abrey LE. Maintenance therapy for central nervous system lymphoma with rituximab. Leuk. Lymphoma 50(9), 1548-1551 (2009).

20. Sehn LH, Donaldson J, Filewich A et al. Rapid infusion rituximab in combination with corticosteroid-containing chemotherapy or as maintenance therapy is well tolerated and can safely be delivered in the community setting. Blood 109(10), 4171-4173 (2007).

21. Dakhil S, Hermann R, Schreeder MT et al. Phase III safety study of rituximab administered as a 90-mininfusion in patients with previously untreated diffuse large B-cell and follicular lymphoma. Leuk. Lymphoma 55(10), 2335-2340 (2014).

- Fosters the US FDA's approval of rapidly infusing rituximab over $90 \mathrm{~min}$ as opposed to standard rate in patients with systemic B-cell lymphomas.

22. Lang D, Prouse J, Barry F et al. Evaluation of the safety and feasibility of rapid rituximab infusion. Asia Pac. J. Clin. Oncol. 8(1), 71-75 (2012).

23. Atay S, Barista I, Gundogdu F, Akgedik K, Arpaci A. Rapid-infusion rituximab in lymphoma treatment: 2-year experience in a single institution. J. Oncol. Pract. 8(3), 141-143 (2012).

24. Patel J, Ho M, Ho V et al. Rapid infusion rituximab for maintenance therapy: is it feasible? Leuk. Res. Treatment 2013, 629283 (2013).

25. National Cancer Institute. Common terminology criteria for adverse events v4.0. (3 March 2017) (2009).www.eortc.be/services/doc/ctc/CTCAE_4.03_2010-06-14_QuickReference_5x7.pdf

26. Salar A, Casao D, Cervera M et al. Rapid infusion of rituximab with or without steroid-containing chemotherapy: 1-yr experience in a single institution. Eur. J. Haematol. 77(4), 338-340 (2006).

27. Lang DS, Keefe DM, Schultz T, Pearson A. Predictors of acute adverse events from rapid rituximab infusion. Support. Care Cancer 21(8), 2315-2320 (2013).

28. Dolecek TA, Propp JM, Stroup NE, Kruchko C. CBTRUS statistical report: primary brain and central nervous system tumors diagnosed in the United States in 2005-2009. Neuro Oncol. 14(Suppl. 5) v1-v49 (2012).

29. Chiang J, Chan A, Shih V, Hee SW, Tao M, Lim ST. A prospective study to evaluate the feasibility and economic benefits of rapid infusion rituximab at an Asian cancer center. Int. J. Hematol. 91(5), 826-830 (2010).

30. Davies A, Merli F, Mihaljevic B et al. Efficacy and safety of subcutaneous rituximab versus intravenous rituximab for first-line treatment of follicular lymphoma (SABRINA): a randomized, open-label, Phase III trial. Lancet Haematol. 4(6), e272-e282 (2017).

31. Larouche JF, Bergeron M, Hampson G, Illidge T, Delage R. Rituximab cerebrospinal fluid levels in patients with primary central nervous system lymphoma treated with intravenous high dose rituximab. Blood 118(21), 714-714 (2011).

32. Mao CP, Brovarney MR, Dabbagh K, Birnbock HF, Richter WF, Del Nagro CJ. Subcutaneous versus intravenous administration of rituximab: pharmacokinetics, CD20 target coverage and B-cell depletion in cynomolgus monkeys. PLoS ONE 8(11), e80533 (2013).

33. Kagan L, Turner MR, Balu-Iyer SV, Mager DE. Subcutaneous absorption of monoclonal antibodies: role of dose, site of injection and injection volume on rituximab pharmacokinetics in rats. Pharm. Res. 29(2), 490-499 (2012). 